\title{
Radiation and inhibition of angiogenesis by canstatin synergize to induce HIF-1 $\alpha-$ mediated tumor apoptotic switch
}

\author{
Claire Magnon, ${ }^{1}$ Paule Opolon, ${ }^{1}$ Marcel Ricard, ${ }^{2}$ Elisabeth Connault, ${ }^{1}$ Patrice Ardouin, ${ }^{3}$ \\ Ariane Galaup, 4,5 Didier Métivier, ${ }^{6}$ Jean-Michel Bidart,7 Stéphane Germain, ${ }^{4,5,8}$ \\ Michel Perricaudet, ${ }^{1,9}$ and Martin Schlumberger ${ }^{2,9}$

\begin{abstract}
CNRS-UMR 8121, Laboratoire de vectorologie et transfert de gènes, "2Département de Médecine Nucléaire and CEA-LRC 29V and ${ }^{3}$ Service Commun d'Expérimentation Animale, Institut Gustave Roussy, Villejuif, France. ${ }^{4}$ INSERM U36, Paris, France. ${ }^{5}$ Collège de France, Paris, France. ${ }^{6}$ INSERM, FRE 2939 and ${ }^{7}$ Département de Biologie clinique, Institut Gustave Roussy, Villejuif, France. ${ }^{8}$ Service d'Hématologie Biologique A, Assistance Publique—Hôpitaux de Paris, Hôpital Européen Georges Pompidou, Paris, France. Université Paris-Sud, Paris, France.
\end{abstract}

\begin{abstract}
Tumor radioresponsiveness depends on endothelial cell death, which leads in turn to tumor hypoxia. Radiation-induced hypoxia was recently shown to trigger tumor radioresistance by activating angiogenesis through hypoxia-inducible factor 1-regulated (HIF-1-regulated) cytokines. We show here that combining targeted radioiodide therapy with angiogenic inhibitors, such as canstatin, enhances direct tumor cell apoptosis, thereby overcoming radio-induced HIF-1-dependent tumor survival pathways in vitro and in vivo. We found that following dual therapy, HIF-1 $\alpha$ increases the activity of the canstatin-induced $\alpha_{v} \beta_{5}$ signaling tumor apoptotic pathway and concomitantly abrogates mitotic checkpoint and tetraploidy triggered by radiation. Apoptosis in conjunction with mitotic catastrophe leads to lethal tumor damage. We discovered that HIF-1 displays a radiosensitizing activity that is highly dependent on treatment modalities by regulating key apoptotic molecular pathways. Our findings therefore support a crucial role for angiogenesis inhibitors in shifting the fate of radiation-induced HIF- $1 \alpha$ activity from hypoxia-induced tumor radioresistance to hypoxia-induced tumor apoptosis. This study provides a basis for developing new biology-based clinically relevant strategies to improve the efficacy of radiation oncology, using HIF-1 as an ally for cancer therapy.
\end{abstract}

\section{Introduction}

Radiation is used to treat a variety of solid tumors. In thyroid cancer patients, radioiodine ${ }^{131}$ I provides a unique and effective system for both the detection of neoplastic foci with ${ }^{131}$ I total body scanning and for the destruction of thyroid cells by delivering lethal doses of radiation $(1,2)$. The sodium iodide symporter (NIS) mediates the active transport of ${ }^{131}$ I into thyroid cells (3-5). A novel therapeutic approach involves inducing a targeted effect of radiation following NIS gene transfer in NIS-defective follicular thyroid or nonthyroidal carcinoma cell lines, allowing accumulation of iodide both in vitro and in vivo (6-11). However, infection of xenografted tumors in mice with an adenovirus encoding NIS (AdNIS) followed by systemic in vivo administration of ${ }^{131}$ I activity consistent with that used in clinical settings, does not induce significant tumor growth inhibition $(6,9)$.

Tumor sensitivity to radiation is dependent on endothelial and tumor cell vulnerability that eventually leads to cell death (12). However, Moeller et al. recently demonstrated that radiation also promotes angiogenesis, thereby inducing radioresistance (13). ROSs generated by irradiated tumor tissue induce hypoxia-inducible factor 1 (HIF-1) activity that in turn induces

Nonstandard abbreviations used: AdNIS, adenovirus encoding NIS; CAIX, carbonic anhydrase IX; CanHSA, canstatin-HSA; DFO, desferrioxamine; HIF-1, hypoxia-inducible factor 1; NIS, sodium iodide symporter; RCC, renal cell carcinoma; TRP-1, tyrosinase related protein-1; VHL, von Hippel-Lindau.

Conflict of interest: C. Magnon was financed by Assistance Publique-Hôpitaux de Paris, the Institut National de la Santé et de la Recherche Médicale, and the

Commissariat à l'Energie Atomique.

Citation for this article: J. Clin. Invest. 117:1844-1855 (2007). doi:10.1172/JCI30269. the expression of angiogenic growth factors such as VEGF or bFGF. These cytokines promote endothelial survival pathways and counteract the radiation-induced apoptosis in both tumor and endothelial cells (13). To circumvent this radiation-induced protective angiogenic response, one promising therapeutic approach may be to combine angiogenesis inhibition with radiation (14-22). During hypoxia, an intricate balance exists between factors that induce and those that counteract apoptosis or even stimulate cell proliferation (23-25). Combined radiation and antiangiogenesis treatment could thus be expected to inhibit the HIF-1-induced angiogenic response. However, HIF-1 has also been shown to induce tumor cell apoptosis (23-25). In the present study, we address the question of whether HIF-1 regulates the balance between adaptation of tumor cells to the hypoxic environment and self-sacrifice by apoptosis.

Here we combined ${ }^{131}$ I radiotherapy with angiogenesis inhibition, using both NIS and canstatin delivered by adenovirus, and studied the role of HIF-1 in the induction of apoptosis in endothelial and tumor cells. Canstatin-HSA (CanHSA), a fragment of human collagen, is known to silence cytokine-induced angiogenesis pathways by binding to $\alpha_{v} \beta_{3}$ - and $\alpha_{v} \beta_{5}$-integrin receptors and triggering mitochondrial apoptosis (26-29).

In the present study, we show that administration of ${ }^{131} \mathrm{I}$, at activities consistent with those used in the clinical setting, combined with an angiogenesis inhibitor strongly impedes growth of both xenografted nonthyroidal tumors and spontaneously occurring tumors in a transgenic mouse model. This dual therapy blocked angiogenesis, induced severe tumor hypoxia, and was followed by greatly enhanced tumor apoptosis. In contrast, treating 
A

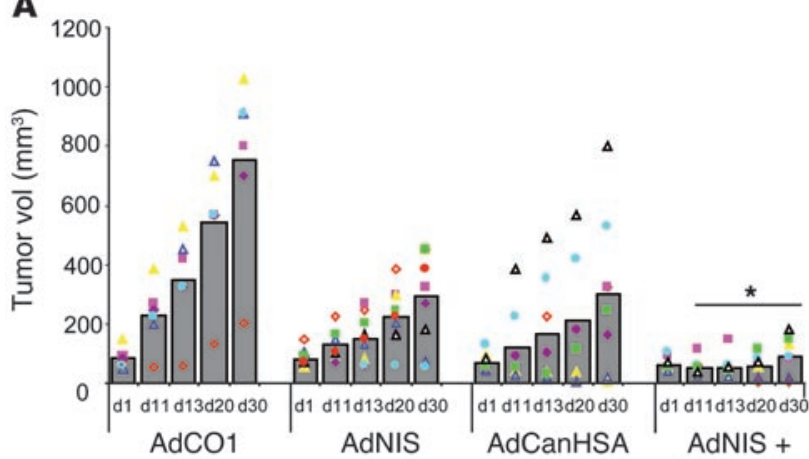

AdCanHSA

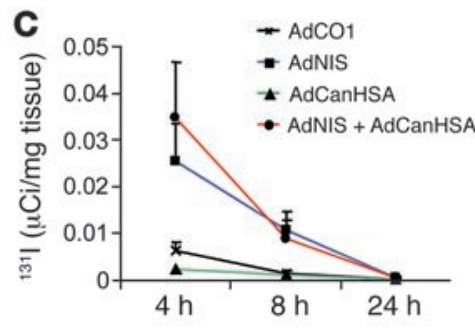

B

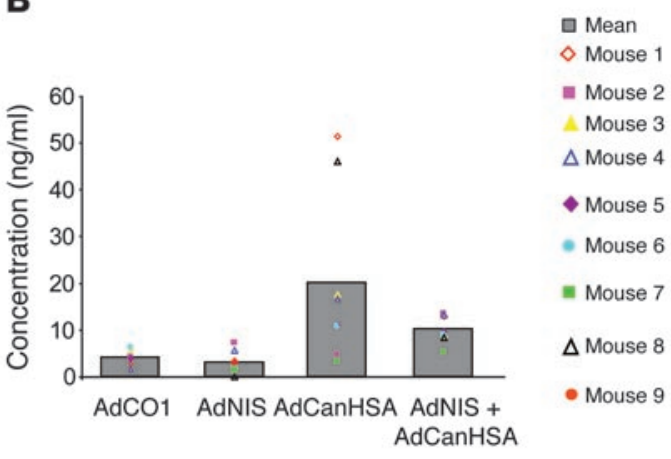

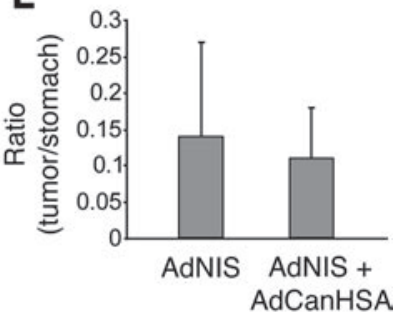

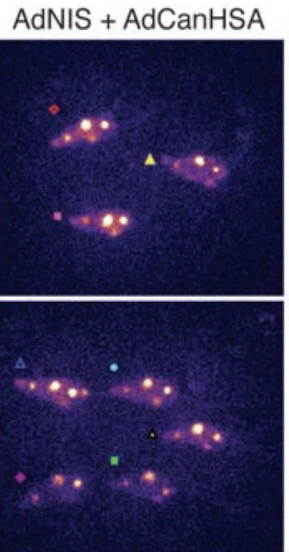

E

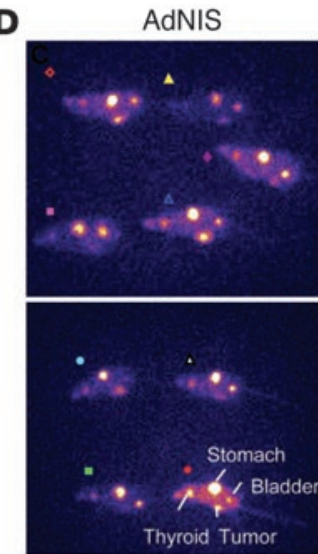

Figure 1

Therapeutic efficiency of AdNIS-131| therapy combined with AdCanHSA in vivo. (A) Growth of AdCO1-, AdNIS-, AdCanHSA-, and AdNIS-AdCanHSAinfected MDA-MB-231 xenografted tumors (2 intratumor injections of the appropriate adenoviruses, 72 hours apart) on nu/nu mice after injection of $300 \mu \mathrm{Ci}$ of ${ }^{131}$ I. Tumor volumes were measured once a week over 4 weeks. Results are mean \pm SEM for 9 mice for each treatment group. Representative of 4 separate experiments. ${ }^{*} P<0.05$. (B) ELISA quantification of CanHSA in the sera of the same mice represented above, at day 4 after injection of iodide. (C) In vivo kinetics of iodide uptake in AdCO1-, AdNIS-, AdCanHSA-, and AdCanHSA-AdNIS-infected MDA-MB-231 tumors ( $n=3$ per group). Infected tumors were removed 4, 8, and 24 hours following injection of ${ }^{131}$ I (300 $\mu$ Ci) i.p. ${ }^{131}$ I was measured using a gamma counter. Results are expressed as the mean number of $\mu \mathrm{Ci}$ per milligram of tumor tissue $\pm \mathrm{SEM}$. (D) In vivo imaging of mice harboring AdNIS- or AdCanHSA-AdNIS-infected MDA-MB-231 xenografts (left flank) 4 hours after i.p. injection of $50 \mu$ Ci of ${ }^{123}$ and 24 hours before ${ }^{131}$ I injection. Images were acquired through scintigraphy over a 10-minute exposure and have an equivalent background. There was also physiological accumulation of iodide in the bladder, stomach, and thyroid gland. Mice were treated with L-thyroxine to avoid massive uptake by the thyroid. (E) In vivo assessment of iodide uptake by AdNIS- or AdCanHSA-AdNIS-infected MDA-MB-231 tumors 4 hours after ${ }^{123}$ injection, as described for D. A fixed region of interest (ROI) was drawn and was imposed on each tumor and on each stomach (as standard uptake). Pixel intensity per ROI was measured with ImageJ software. Results are expressed as the ratio (tumor/stomach) between the maximal pixel intensity in the 2 ROIs.

tumors by single therapies led to tumor resistance by enhancing HIF-1-mediated endothelial cell survival. We further identified the HIF-1 signaling molecular mechanism by which damage to endothelial cells following combined therapy results in increased tumor cell death. In vitro studies showed that HIF-1 activity plays a crucial role in: (a) controlling the canstatin-induced mitochondrial apoptotic pathway mediated by binding to $\alpha_{v} \beta_{5}$ integrin in MDA-MB-231 tumor cells, whereas endothelial cells underwent apoptosis in response to canstatin in the absence of HIF-1; (b) controlling the mitosis checkpoint and tetraploid status of tumor and endothelial cells following radiation, sensitizing cells to death by mitotic catastrophe; and subsequently (c) increasing the number of apoptotic tumor cells treated with both canstatin and radiation, which was validated using HIF-1 siRNA.

We therefore propose that HIF-1 signaling activation has important implications for optimizing targeted therapeutic schedules in trials aimed at combining angiogenesis inhibitors and radiation.

\section{Results}

In vivo therapentic efficiency of the AdCanHSA-AdNIS-131I combination on the mammary tumor model. AdNIS-treated tumors exhibit specific uptake of radioactive iodide (9). However, the activities of ${ }^{131} \mathrm{I}$ (1-16 $\mathrm{mCi}$ per dose) that have to be administered to mice bearing NIS-transduced xenografted tumors in order for a therapeutic effect to be observed are far too high to be applicable in the clinic $(10,30)$. To improve the efficiency of AdNIS- ${ }^{131}$ I treatment, we investigated here the antitumor potential of radiation combined with an angiogenesis inhibitor for 4 weeks (16). Following preliminary experiments aimed at determining the minimal dose of radiation needed to induce a suboptimal antitumor effect following AdNIS infection, we used $300 \mu \mathrm{Ci}$ of ${ }^{131} \mathrm{I}$ in experiments performed thereafter (data not shown).

Interestingly, treatment of preestablished mammary MDAMB-231 xenografted tumors with the AdCanHSA-AdNIS- ${ }^{131}$ I combination induced a very early tumor growth inhibition com- 
A

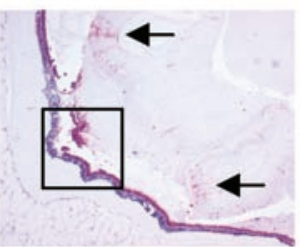

B

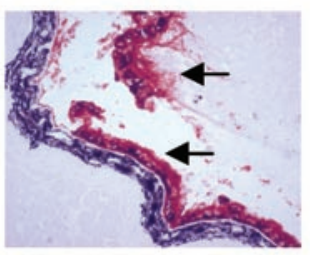

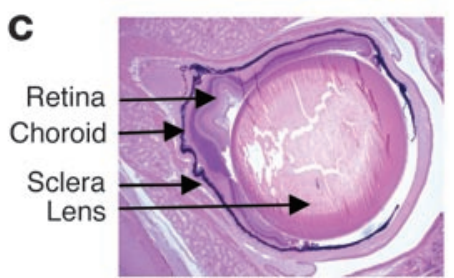

AdNIS + AdCanHSA

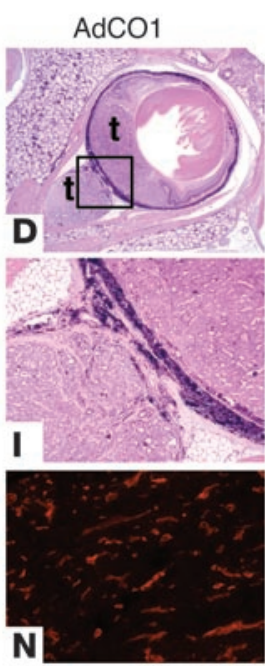

S
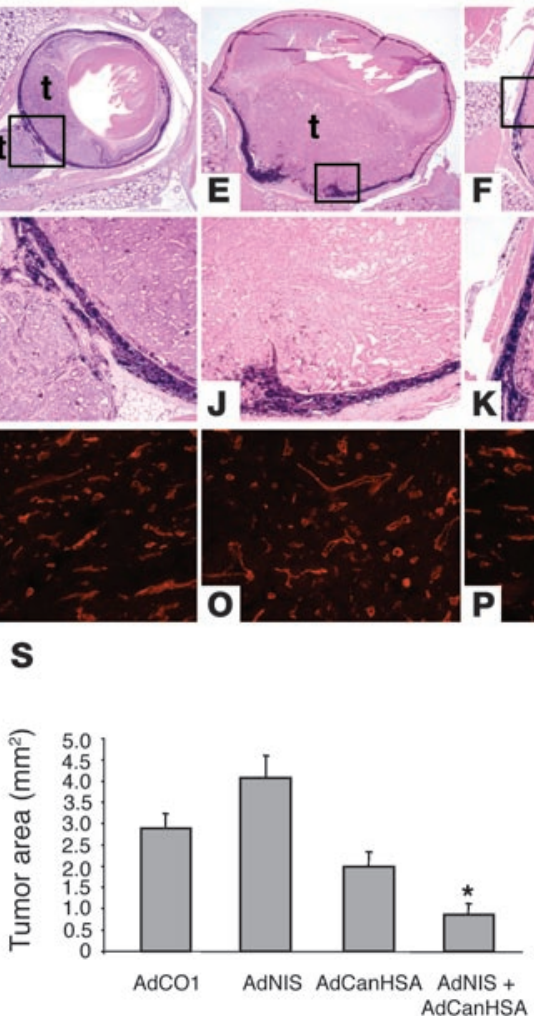

$F$
AdCanHSA
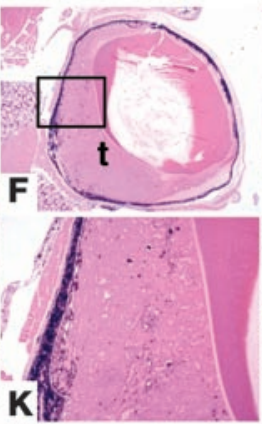

L
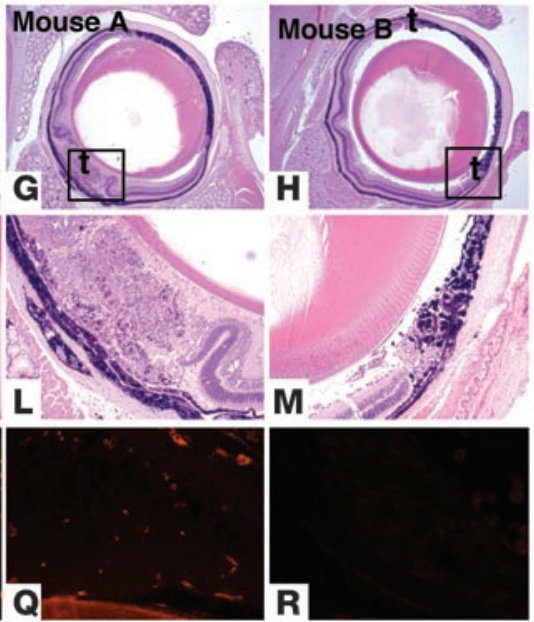

T

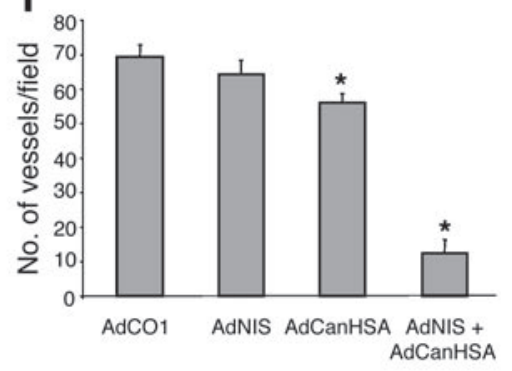

Figure 2

Combined effects of AdNIS-131I therapy with AdCanHSA in transgenic TRP-1 mouse model. (A) Immunohistological analysis of AdNIS-infected RPE tumor with an anti-NIS polyclonal antibody (original magnification, $\times 50$ ). (B) Higher-magnification of boxed area in $\mathbf{A}$ (original magnification, $\times 200$ ). Positive cells were located in the RPE tumor and in the retina (arrows). (C) Representative nontumor eye section with location of retina, choroid, sclera, and lens (arrows). (D-H) Histology of the RPE tumor 45 days after birth. Representative transgenic eye section treated with 2 systemic injections of $\mathrm{Ad}_{\mathrm{x}}$ and a single intraorbital injection of $\mathrm{Ad}$, and a single ${ }^{131}$ I injection of $300 \mu \mathrm{Ci}$. AdCO1 and AdCO1 (D); AdCO1 and AdNIS (E); AdCanHSA and AdCO1 (F); or AdCanHSA and AdNIS (G and H) (original magnification, $\times 25$ ). Note that AdCO1-treated tumor cells grew consistently and occupied half of the eyeball when mice were 45 days old (D). In contrast, only a few tumor cells persisted in AdCanHSAAdNIS-treated eyeball (H). t, tumor. (I-M) Higher-magnification images of areas in black boxes in D-H, respectively (original magnification, $\times 100$ ). (S) Measurement of tumor areas in the posterior eyeball of each TRP-1 transgenic mouse treated with the appropriate adenoviruses. Results are the mean \pm SEM $(n=10)$. ( $\mathbf{N}-\mathbf{R})$ Assessment of intratumor vascularization using lectin immunostaining after each treatment described above (original magnification, $\times 100$ ). AdCO1 and AdCO1 (N); AdCO1 and AdNIS (0); AdCanHSA and AdCO1 (P); AdCanHSA and AdNIS (Q and R) (same as in black boxes in $\mathbf{D}-\mathbf{H}$ ). Original magnification, $\times 200$. (T) Mean number of intratumor vessels for each group \pm SEM. ${ }^{*} P<0.05$.

pared with that seen in control groups ( $86 \%$ as compared with the AdCO 1 group; $P<10^{-4}$ ). Two of the 8 tumors became undetectable, indicating complete regression following a single administration of ${ }^{131} \mathrm{I}$ (Figure 1A).

To determine whether the antitumor effect was related to coexpression of NIS and CanHSA, expression of both proteins was investigated in vivo. As expected, CanHSA was strongly expressed in the sera of mice infected by AdCanHSA or AdCanHSA combined with AdNIS (Figure 1B). Moreover, CanHSA did not impede tumor ${ }^{131}$ I uptake after injection of $300 \mu \mathrm{Ci}^{131}$ I (Figure 1C): at 4 or 8 hours following iodide injection, no quantitative difference in iodide uptake, measured ex vivo, was observed between tumors infected by AdNIS-131I alone or AdCanHSA-AdNIS-131I $(P=0.29)$. In tumors infected with AdCanHSA alone or AdCO1 as a control, no ${ }^{131}$ I uptake was found $(P<0.05)$. In vivo, scintigraphy was carried out for animals treated with AdNIS. No difference in iodide uptake was observed between AdNIS- ${ }^{131}$ I - and AdCanHSA-AdNIS${ }^{131}$ I-treated tumors $(P=0.3)$ (Figure 1, D and E). Therefore, iodide uptake and retention time were not altered in AdNIS-infected tumors after CanHSA expression. 
Invivo AdCanHSA-AdNIS- ${ }^{131}$ I combined therapy on the transgenicretinal pigmented epithelium tumor model. Transgenic tyrosinase related protein-1 (TRP-1) mice developing intraocular tumors were injected intravenously with AdCanHSA or AdCO1 and treated with intraorbital injection of AdNIS or AdCO1. AdNIS drove local and specific expression of a protein within the choroid, the retina, and the tumor (Figure 2, A and B). AdNIS-infected TRP-1 intraocular tumors were injected i.p. with ${ }^{131} \mathrm{I}$, and radioiodide uptake was quantified 4 hours later $(1.35 \% / \mathrm{g}$ ocular tissue; $P=0.005$ compared with AdCO1 group), confirming specific iodide uptake in the eyeballs and the specificity of the AdNIS${ }^{131}$ I antitumor effect described below.

Tumor growth was inhibited by AdCanHSA-AdNIS-131 I combined therapy (mean, $0.8 \mathrm{~mm}^{2}$ compared with AdCO1 treatment, $2.9 \mathrm{~mm}^{2}$; $70 \%$ tumor growth inhibition; $P=0.001)$. These results were also significant when compared with treatment with AdNIS-131I alone (mean, $4.1 \mathrm{~mm}^{2} ; P=10^{-5}$ ) or AdCanHSA-131 I alone (mean, $2 \mathrm{~mm}^{2} ; P=0.009$ ) (Figure 2, D-M and S).

We next assessed whether angiogenesis was also inhibited by combined treatment in this model using lectin immunostaining. As shown in Figure 2, N-P, numerous vessels remained with an open lumen in AdCO1-, AdNIS- ${ }^{131}$ I- and AdCanHSA- ${ }^{131}$ I-treated tumors as compared with AdCanHSA-AdNIS- ${ }^{131}$ I-treated tumors (Figure 2, Q and R). Quantification of the intratumor vascularization showed a strong antiangiogenic effect only in the AdCanHSA-AdNIS- ${ }^{131}$ I- $(81 \% ; P<0.001)$ and a slight one in the AdCanHSA- ${ }^{131}$ I-treated $(19 \% ; P=0.002)$ as compared with the AdCO1-treated group (Figure 2T).

In conclusion, ${ }^{131}$ I combined therapy cured different local lesions and was not found to be tumor type dependent.

In vivo AdCanHSA-AdNIS-131 I combined therapy triggers both angiogenesis inhibition with concomitant abrogation of HIF-1 expression and tumor mitochondrial apoptosis. Radiation has recently been shown to induce preferential apoptosis of vascular endothelial cells and to increase HIF-1 activity, leading to secretion of proangiogenic molecules that regulate angiogenesis-dependent tumor progression $(12,13)$. Paradoxically, HIF-1 can also drive apoptosis $(23,25)$. To further explain the antitumor effect of the dual therapy described above, we investigated the intratumor angiogenic network, HIF-1 expression, and apoptotic signaling pathways in response to radiation and/or canstatin.

To confirm the potential link between angiogenesis and MDAMB-231 tumor progression represented in Figure 1A, we assessed intratumor microvessels using CD34 immunostaining. Although vasculature of AdNIS-131I- or AdCanHSA-131I-treated tumors was slightly inhibited at day 15 , vessel development was highly stimulated at day 30 (60.8 and 68.2 vessels $/ \mathrm{mm}^{2}$, respectively, at day 30) (Figure 3A). In contrast, intratumor vessel development was steadily inhibited in AdCanHSA-AdNIS-131I-treated tumors at days 15 and 30 (29.5 vessels $/ \mathrm{mm}^{2}$ as compared with 58 vessels $/ \mathrm{mm}^{2}$ in the AdCO1-treated group; $P=0.004$, at day 30) (Figure $3 \mathrm{~A}$ ), which is consistent with the constant tumor growth inhibition described in Figure 1A.

HIF-1 is known to stimulate tumor angiogenesis $(13,23,24)$. As expected, AdNIS-131 I or AdCanHSA- ${ }^{131}$ I alone induced long-lasting expression of carbonic anhydrase IX (CAIX), an HIF-1-responsive gene (31) (Figure 3, C and D). At day 30, angiogenesis induced by radiation or canstatin alone was subsequent to HIF-1 expression. Interestingly, AdCanHSA-AdNIS- ${ }^{131}$ I combined therapy-induced expression of CAIX at day 15 was unexpectedly abrogated over the treatment period (5-fold decrease in area fraction of CAIX-stained cells between days 15 and 30). We further showed that at day 30 CAIX protein expression mirrored HIF-1 mRNA expression. Additionally, in situ hybridizations showed that combined therapy did not elicit HIF-1 mRNA expression over the treatment period (day 30), whereas AdCanHSA-131 I or AdNIS- ${ }^{131}$ I alone did $(P=0.03)$ (Figure 3E). Accordingly, at day 30, HIF-1 protein expression levels were not also detected by Western blotting following AdCanHSAAdNIS-131I treatment, as compared with radiation alone or canstatin alone (Figure $3 \mathrm{~F}$ ).

These results indicate that radiation alone or canstatin alone both induce a mechanism of resistance by stimulating the HIF-1dependent survival angiogenic pathways that correct for oxygen imbalances. Conversely, the combined therapy might decrease overall tumor resistance to radiation.

We next showed that AdCanHSA-AdNIS- ${ }^{131}$ I combined therapy combined therapy was the only therapeutic approach resulting in marked and constant apoptosis (9- and 14-fold increase in apoptotic MDA-MB-231 cells as compared with the AdCO1-treated group, at days 15 and 30 after infection, respectively; $P<0.0001$ ) (Figure 3G). More precisely, the AdCanHSA-AdNIS-131I-induced apoptosis was secondary to mitochondrial damage, leading to both caspase- 9 and caspase-3 cleavage (Figure 3, H and I). In contrast, AdCanHSA-131I and AdNIS-131I alone induced a weaker proapoptotic signal, at day 15 , which faded out at day 30 (5- and 2 -fold increase in apoptotic cells/field as compared with AdCO1 group $\left[P<10^{-3}\right]$ at days 15 and 30, respectively) (Figure $3 \mathrm{G}$ ).

This experiment allowed us to show that combining the treatments induced a marked decline in angiogenesis with concomitant abolition of HIF-1 and CAIX expression and potent apoptosis. Alternatively, HIF-1 has been shown to induce apoptosis (23-25). The extinction of HIF-1 expression in AdCanHSA-AdNIS${ }^{131}$ I-treated tumors might thus be a consequence of induction of an HIF-1-dependent apoptotic process.

To further characterize a possible link between HIF-1 and a tumor cell death pathway following administration of combined therapy, we explored in vitro the possibility that HIF-1 participates in the control of mitochondrial apoptosis and mitotic checkpoint after cell exposure to canstatin alone, radiation alone, or combined therapy.

In vitro, HIF-1 and canstatin activate the integrin-mediated mitochondrial apoptotic signaling pathway. To investigate a possible role of HIF-1 in mitochondrial tumor apoptotic cell death following administration of canstatin, we performed in vitro studies of HIF-1 expression and caspase- 3 cleavage from AdCanHSA-infected MDA-MB-231 tumor cells. Treatment of MDA-MB-231 tumor cells with $100 \mu \mathrm{M}$ desferrioxamine (DFO) potently induced HIF-1 stabilization.

In vivo, canstatin combined with radiation downregulated the expression of HIF-1 (Figure 3C). As expected, HIF-1 protein expression was also abolished by CanHSA in vitro (Figure 4A, right panel). This phenomenon was accompanied by caspase- 3 cleavage. In contrast, CanHSA induced a low apoptotic effect in tumor cells that did not express HIF-1 (Figure 4A, compare center and right panels). Both HIF-1 expression inhibition and increased cleavage of caspase-3 depend on the level of CanHSA infection (Figure 4B, right panel). The results shown in Figure $4 \mathrm{C}$ further indicated that CanHSA treatment was able to mediate a cytotoxic effect only when HIF-1 accumulation was high.

We confirmed the link between HIF-1 and the induction of a tumor apoptotic pathway by CanHSA using the von Hippel-Lindau-defective (VHL-defective) renal cell carcinoma 4 (RCC4) cell 
A
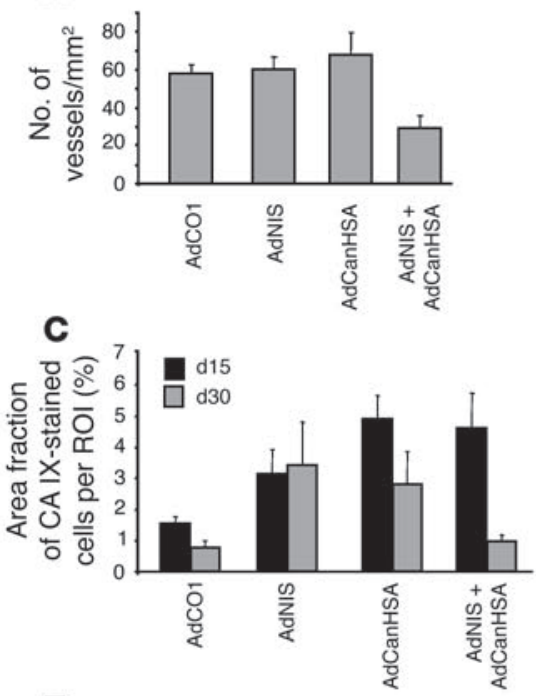

E

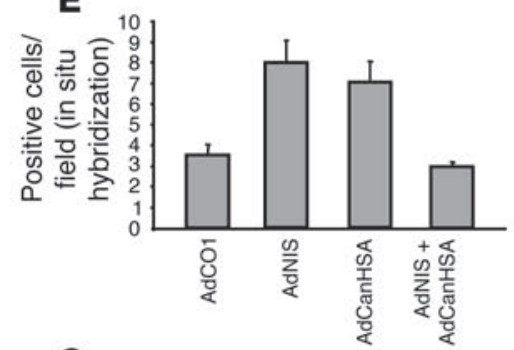

G

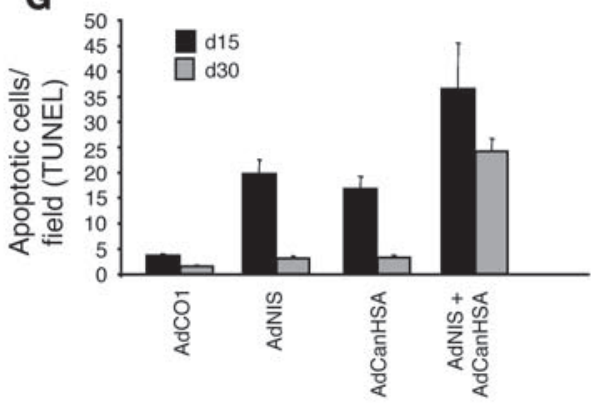

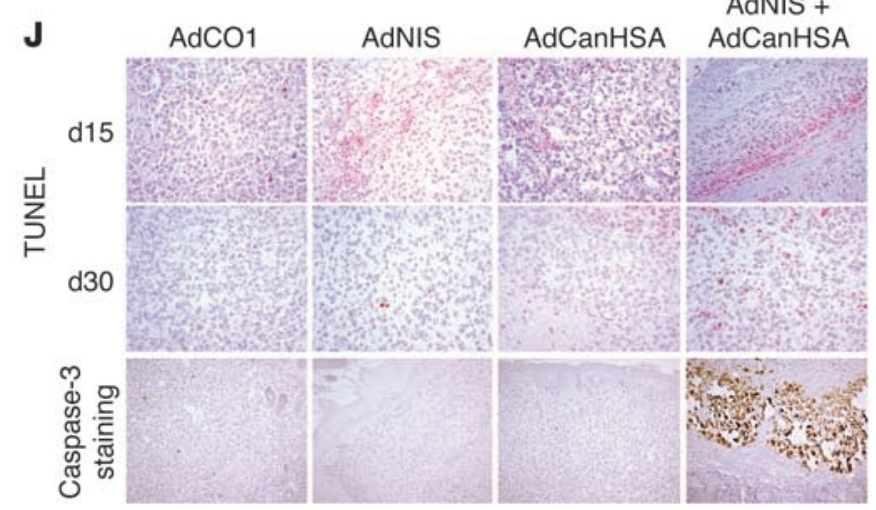

D

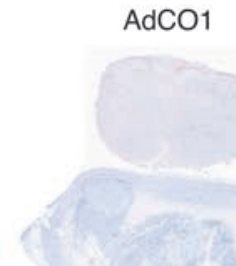

B

d30

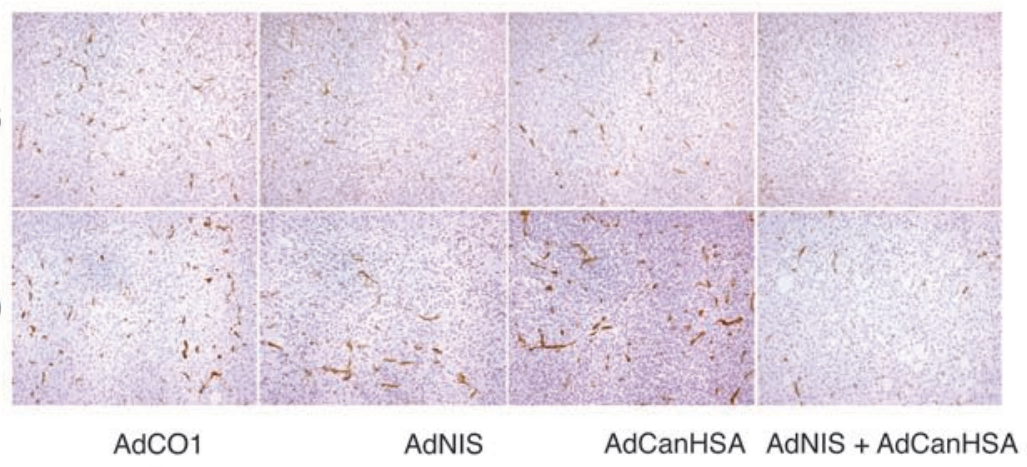

$\mathbf{F}$

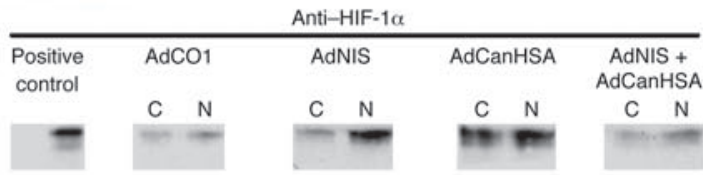

H

I

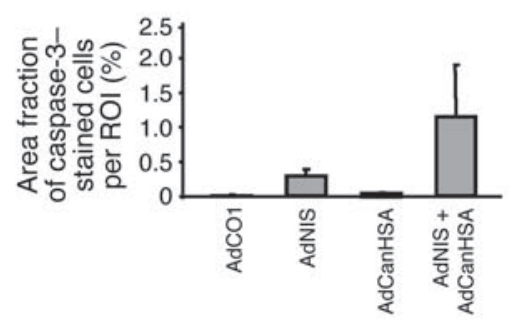




\section{Figure 3}

In vivo, detection of hypoxic, apoptotic, and endothelial markers in MDA-MB-231 xenografted tumors following AdNIS-131/ therapy combined with AdCanHSA. (A) The number of CD34-stained intratumor microvessels per square millimeter was quantified by digital analysis of tumor tissue sections at day 30 after infection. Original magnification, $\times 100$. (B) Representative images of CD34 endothelial immunostaining. (C) Quantification of the area fraction of hypoxic CAIX-stained cells within digitized sections from AdCO1-, AdNIS-, AdCanHSA-, and AdCanHSAAdNIS-injected tumors at days 15 and 30 (i.e., at days 8 and 20 after injection of ${ }^{131}$ I). (D) Representative CAIX-stained digitized sections for each group. (E) Quantification of HIF-1 mRNA levels by in situ hybridization analysis. The number of cells per field producing mRNA HIF-1 $\alpha$ was quantified within tumors sections $(\times 40)$ at day 30 following injections of appropriate adenoviruses listed above. (F) Western blotting analysis of HIF-1 $\alpha$ protein levels within nuclear $(\mathrm{N})$ and cytoplasmic $(\mathrm{C})$ extracts of adenovirus-infected xenografted tumors. A whole-cell lysate of RCC (VHL mutated) cells was considered as positive control. Note that HIF-1 $\alpha$ and CAIX have a similar expression pattern at day 30 . (G) The proportion of apoptotic cells per field was quantified within sections (×200) (TUNEL method) at days 15 and 30 after infection. Quantification of the area fraction of caspase-9- (H) and caspase-3-stained cells (I) within digitized sections from treated tumors at day 30 as described in Figure 1 A. (J) Representative illustrations of both TUNEL and caspase-3 staining. Columns, mean \pm SEM of 9 mice for each treated group. All digitized paraffin-embedded tumor sections were obtained from the experiment described in Figure 1A. Original magnification, $\times 200$.

line, which fails to produce a WT VHL protein (VHL mutated), leading to constitutive HIF- $1 \alpha$ overexpression. In these cells, CanHSA induced apoptosis through caspase-3 cleavage (Figure 4D). In contrast, reexpression of WT VHL led to downregulation of HIF-1. Pretreatment of these cells with 50 or $100 \mu \mathrm{M}$ DFO was able to induce HIF-1 expression. In this context, CanHSA also induced caspase-3 cleavage, as observed in MDA-MB-231 cells (Figure 4E).

In contrast, CanHSA-induced apoptosis also occurred independently of HIF-1 in endothelial cells. Indeed, CanHSA-mediated caspase- 3 cleavage was observed without DFO. Induction of HIF-1 protein expression amplified caspase- 3 cleavage but was not essential (Figure 4F).

We then asked whether the extinction of HIF-1 protein expression is due to a canstatin-mediated transcriptional downregulation of HIF-1. In vitro, the expression level of HIF-1 mRNA, evaluated by RT-PCR in tumor and endothelial cells, was not modulated by CanHSA (data not shown).

Taken together, the results indicate that the apoptotic process observed in the in vivo experiments described above was not due to canstatin-induced inhibition of the HIF-dependent survival pathway. We hypothesize that CanHSA-mediated endothelial cell death might induce in vivo HIF-1 protein expression, which can in turn increase the efficacy of CanHSA antiangiogenic therapy by upregulating a tumor apoptotic pathway.

Furthermore, we have previously established that CanHSA sensitivity can be predicted based on integrin gene expression profiles. Indeed, interaction of CanHSA with $\alpha_{v} \beta_{3}$ and $\alpha_{v} \beta_{5}$ integrins selectively triggers the mitochondrial apoptotic pathway (29). We therefore investigated whether $\alpha_{v} \beta_{5}$ integrins mediate the HIF-1dependent tumor apoptotic signal for CanHSA. FACS analysis allowed us to characterize $\alpha_{v} \beta_{5}$ integrin expression on the surface of MDA-MB-231 cells. As shown in Figure 4G, expression of $\alpha_{v} \beta_{5}$ on tumor cells was increased 3-fold following HIF-1 expression $(P<0.01)$. Transfection of an siRNA specifically silencing the $\beta_{5}$ integrin subunit reduced surface expression of $\alpha_{v} \beta_{5}$ by $30 \%(P<0.01)$. This level of inhibition of $\alpha_{v} \beta_{5}$ expression was sufficient to significantly reduce the ability of CanHSA both to reduce HIF-1 protein level and to cleave caspase-3 (Figure $4 \mathrm{H}$ ). In contrast, inhibition of $\alpha_{v} \beta_{3}$ expression in MDA-MB-231 tumor cells using a specific siRNA did not modify the caspase-3 cleavage according to an HIF-1dependent tumor apoptotic process as previously described for $\alpha_{v} \beta_{5}$ integrin (Figure 4I).

To definitively confirm whether HIF-1 is required to induce the CanHSA-induced apoptotic process, an siRNA silencing HIF-1 expression (Figure 6) was transfected into MDA-MB-231 cells.
As shown in Figure 4I, CanHSA-induced cleaved caspase-3 level decreased when HIF-1 expression was downregulated.

Together, our results show that both $\alpha_{v} \beta_{5}$ integrins and HIF-1 play pivotal roles in stimulating the CanHSA-induced mitochondrial tumor apoptotic pathway.

In vitro, HIF-1 and radiation control the $G_{2} / M$ checkpoint, tetraploidy, and bypoploidy. We next investigated whether HIF-1 might be involved in the control of both apoptosis and genomic instability following irradiation of tumor and endothelial cells. Cell-cycle arrest in mitosis $\left(\mathrm{G}_{2} / \mathrm{M}\right.$ checkpoint) serves to repair DNA damage caused by ionizing radiation. Defects in the mitotic checkpoint generate tetraploid and/ or aneuploid states that contribute to tumorigenesis and acquired resistance to some therapeutics, especially in the absence of functional p53 $(32,33)$. If the $\mathrm{G}_{2} / \mathrm{M}$ checkpoint is abolished, cells can enter mitosis prematurely, before DNA damage has been repaired. Aberrant mitoses sensitize cells to death by mitotic catastrophe (34).

Here, irradiation of p53-null MDA-MB-231 tumor cells induced a dose-dependent defective mitotic checkpoint, promoting tetraploidy without signs of hypoploidy ( $\mathrm{subG}_{1}$ ) (Figure 5, A and B). HIF-1 expression abolished both mitotic checkpoint and tetraploidy and led DFO-treated MDA-MB-231 tumor cells to enter mitosis after irradiation at different doses (Figure 5, A and B).

In irradiated p53-mutated RCC (VHL mutated) cells, stabilization of HIF-1 expression inhibited cell-cycle arrest defects, thereby preventing cell tetraploidy, as compared with RCC cells (VHL WT), which do not express HIF-1 (Figure 5, C and D).

Irradiated p53 WT HUVECs underwent cell-cycle arrest in $\mathrm{G}_{2} / \mathrm{M}$. Apoptosis $\left(\mathrm{subG}_{1}\right)$ was also rapidly initiated. Cells were capable of recovering mitosis and eliminating tetraploid cells when HIF-1 expression was induced, as described above (Figure 5, E and F). Furthermore, abrogation of the $\mathrm{G}_{2} / \mathrm{M}$ arrest by HIF-1 increased the hypoploidy, thereby providing a shift from mitosis to apoptosis (35) (Figure 5, E and F). Collectively, our results indicate that HIF-1 plays a crucial role in forcing both endothelial and tumor cells to enter into aberrant mitoses, leading to death (34).

In vitro, HIF-1 triggers the tumor apoptotic switch in response to combined therapy. Having shown that HIF-1 activation is a driving force that shifts cells toward apoptosis, the survival and number of annexin V-positive MDA-MB-231 tumor cells, DFO-treated and nontreated, was analyzed following AdCanHSA infection either alone or in combination with $\gamma$-irradiation. Tumor apoptosis was markedly increased in response to combined therapy only in DFOtreated cells, i.e., when HIF-1 was expressed, as compared with single therapies (53\% of annexin $\mathrm{V}$-positive DFO-treated, $P<0.003$; $34 \%$ and $75 \%$ annexin $V$-negative DFO-treated and -nontreated 


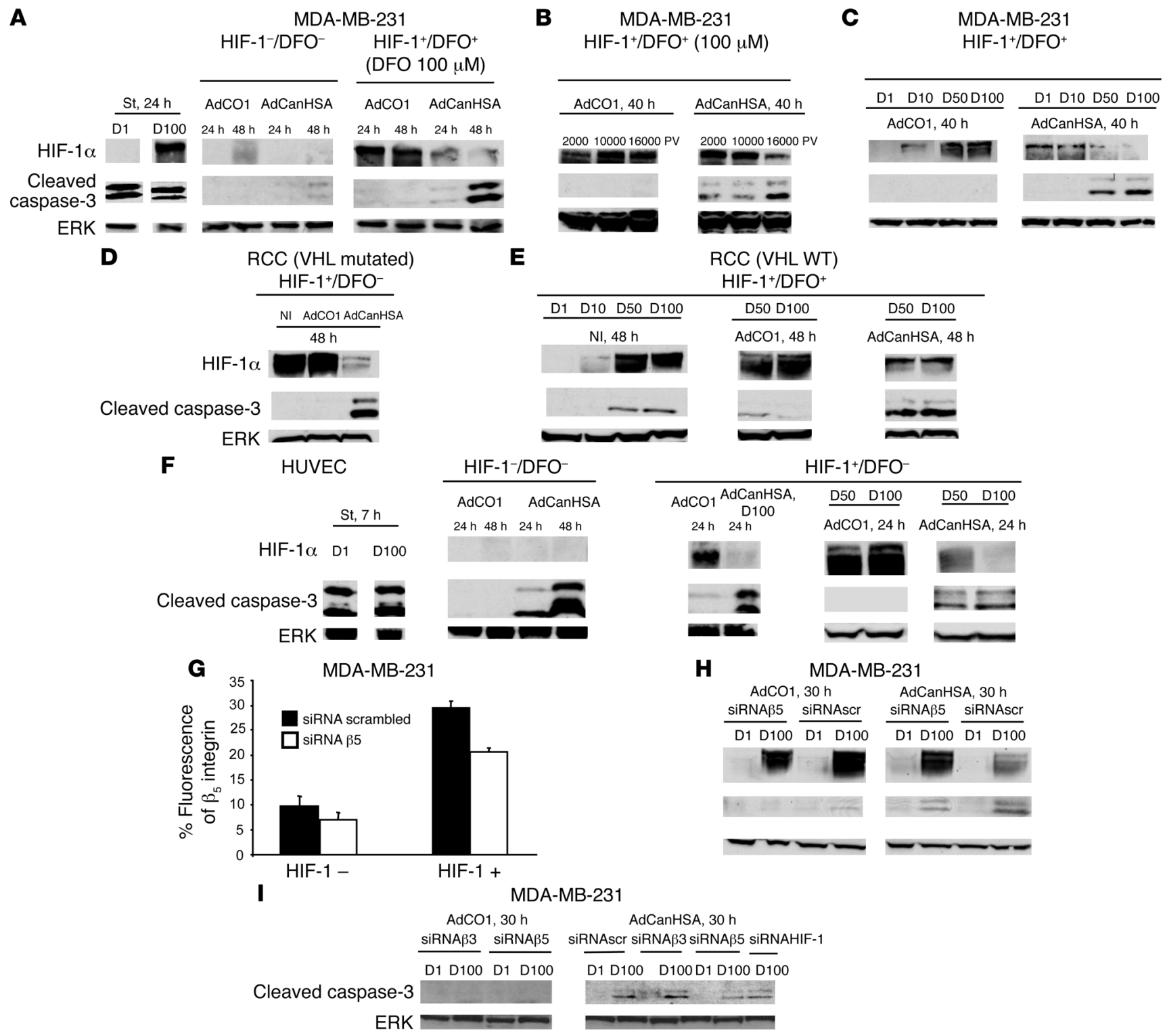

Figure 4

HIF-1 is required for potentiation of integrin-mediated tumor apoptotic signaling pathway induced by CanHSA. The expression levels of both HIF- $1 \alpha$ and caspase-3 were assessed by Western blotting of whole-cell extracts prepared from AdCO1- or AdCanHSA-infected MDA-MB-231 cells, in the presence or absence of DFO (A) at different time points following infection (B) with several MOls $(2,000,10,000$, or 16,000 particle viral [PV]) or (C) at different concentrations of DFO (1, 10, 50, and $100 \mu \mathrm{M}$ [D1, D10, D50, and D100]). Immunoblots of whole-cell extracts, from noninfected (NI) or AdCO1- or AdCanHSA-infected RCC cells expressing VHL mutated (D) or VHL WT (E) in the presence or absence of DFO, confirmed that HIF-1 expression potentiates caspase-3 cleavage following infection with AdCanHSA. (F) Western blotting of protein extracts from HUVECs showed that HIF-1 $\alpha$ is not required to induce the CanHSA-mediated apoptotic process in endothelial cells. Note that HIF-1 $\alpha$ expression slightly accelerated caspase-3 cleavage. Staurosporine (St), a known inducer of caspase-dependent apoptosis, was used as a positive control. To determine whether $\alpha_{v} \beta_{5}$ integrins promote the CanHSA-induced HIF-1 $\alpha$ signaling tumor apoptotic pathway, expression of $\alpha_{v} \beta_{5}$ was silenced through siRNA $\beta_{5}$ on MDA-MB-231 cells expressing or not expressing HIF-1 $\alpha$ (FACS analysis as previously described; ref. 27) (G) and Western blotting was done on whole-cell extracts from AdCO1- or AdCanHSA-infected MDA-MB-231 cells, which were transfected with random siRNA (siRNA scrambled) or siRNA $\beta_{5}(\mathbf{H})$. (I) In parallel, to investigate whether $\alpha_{v} \beta_{3}$ integrins take part in the HIF-1-dependent tumor apoptotic process, MDA-MB-231 cells were also transfected with siRNA $\beta_{3}$ and then infected with AdCO1 and AdCanHSA. Each experiment was done twice. To confirm whether CanHSA triggers an HIF-1-dependent apoptotic process, we transfected an siRNA HIF-1 within the MDA-MB-231 cells.

cells (survival fraction), respectively, $P=0.0008$ ) (Figure 6, A-C). As previously reported, HIF-1 expression enhanced tumor cell death induced by CanHSA alone $(21 \%$ and $12 \%$ of annexin $V$-positive cells, DFO-treated and untreated, respectively; $P=0.01$ ).
To further determine whether expression of HIF-1 controls the tumor apoptotic switch following the combined therapy, an siRNA that specifically silenced HIF-1 expression was used in MDA-MB-231 cells (Figure 6D). As shown in Figure 6, E and F, 


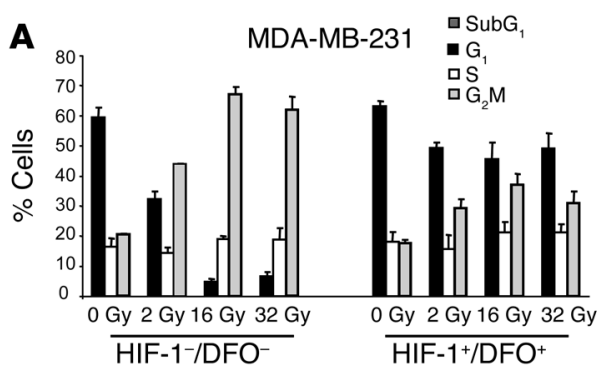

C

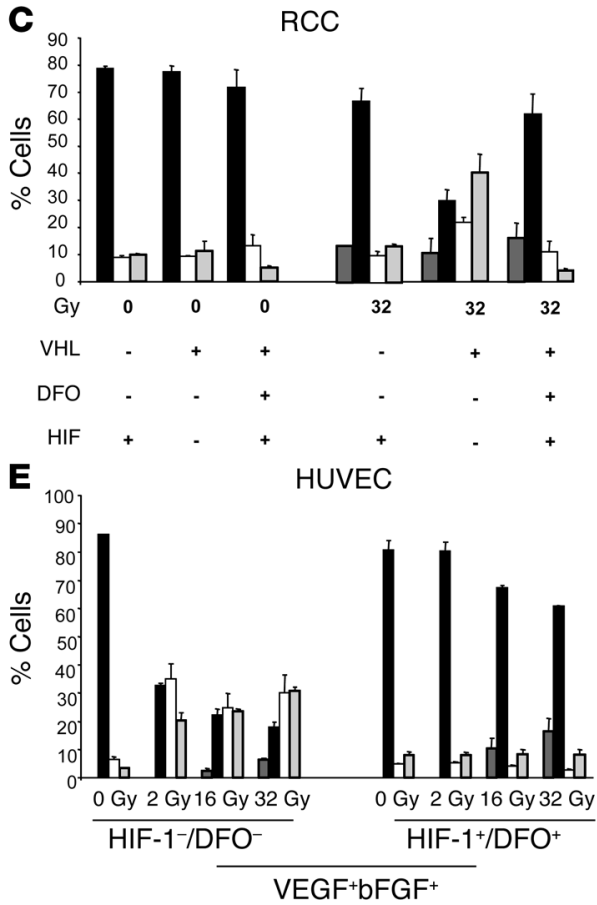

B

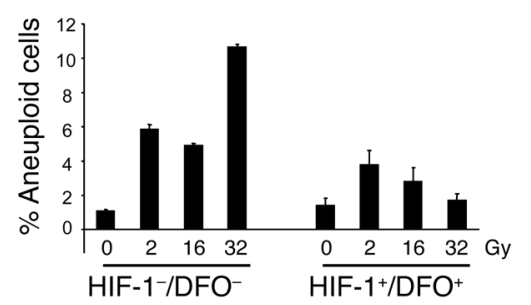

D RCC
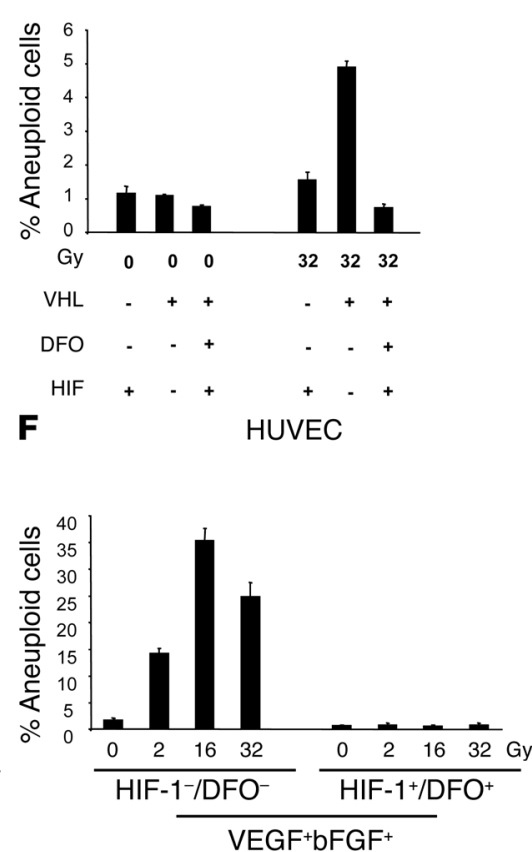

\section{Figure 5}

HIF-1 mediates the control of mitosis checkpoint, aneuploidy, and haploploidy following radiation. Cell-cycle distribution of cells exposed to escalating doses of $\gamma$-radiation (2, 16 , and 32 Gy, for each dose, twice, 3 hours apart). Percentage of cells of the population found in $G_{1}, S, G_{2} / M$ phase and tetraploidy for MDA-MB-231 cells (A and $\mathbf{B}), \mathrm{RCC}$ cells (VHL mutated or WT) (C and D), and HUVECs (E and F). Bars indicate SEM. Each experiment was done twice. All experiments were performed with VEGF and bFGF, confirming that HIF-1 controls this checkpoint in a growth factor-independent manner. inhibition of HIF-1 expression significantly reduced cell death following CanHSA treatment alone or combined therapy (35\% and $9 \%$ of annexin V-positive DFO-treated cells transfected with random siRNA and siRNA HIF-1, respectively; $P<0.05$ ), thus indicating that HIF-1 plays a crucial role in potentiating radiation, confirming in vivo observations.

\section{Discussion}

The efficacy of ${ }^{131}$ I therapy depends on the radiation dose delivered to NIS-transduced tumor tissues, which is related to both ${ }^{131} \mathrm{I}$ uptake and ${ }^{131}$ I retention in tumor cells. In order to compensate for the rapid iodide efflux of nonthyroidal nonorganifying tumor cells, high ${ }^{131}$ I activity (1-16 mCi per dose) must be administered to mice in NIS-transduced xenografted tumors in order for any therapeutic effect to be observed $(10,30)$. However, administering efficient but safe ${ }^{131} \mathrm{I}$ doses is a crucial step toward the clinical application of nonthyroidal radioiodine therapy following NIS gene delivery.

This is the first study to our knowledge to show that cotransferring NIS and CanHSA in vivo within well-established mammary xenografts combined with a single and relatively low ${ }^{131} \mathrm{I}(300 \mu \mathrm{Ci})$ activity leads to a strong tumor size reduction (86\%) and complete eradication in $25 \%$ of cases. It is noteworthy that treatment of $n u / n u$ mice began when each mouse had a large, vascularized solid tumor (i.e., volume $>70 \mathrm{~mm}^{3}$ ) in this study. We have previously shown that CanHSA alone can lead to $98 \%$ tumor growth inhibition ( $20 \%$ of total regression) at an earlier stage of cancer with an immature vascularization (i.e., mean tumor volume of $30 \mathrm{~mm}^{3}$ ) (29). In the present study, only 55\% inhibition of growth of large tumors occurred following CanHSA injection alone, showing that antiangiogenic treatment alone is more efficient for inhibiting small tumor development, by antagonizing endothelial cell growth during the early stage of tumor angiogenesis $(17,36)$. We show here that the combined strategy promotes inhibition and regression of large tumors. To validate these results in tumors developing de novo in their natural tissue environment, we further applied this combined therapy to treat mice of the transgenic TRP-1 model in which ocular tumor progresses through multiple stages in their natural tissue microenvironments, thereby more closely mimicking human cancers (36). This approach confirmed that combined AdCanHSA-AdNIS- ${ }^{131}$ I therapy appears to be a promising therapeutic strategy, although clinical translation of this approach awaits extensive investigation to evaluate potential toxicity. Nevertheless, the concept of combining an antiangiogenic agent with ${ }^{131}$ I therapy may also be valid in patients with thyroid cancer in whom radioiodide is ineffective because the radiation dose is too low (37). This strategy may also be applicable to other human tumors such as pheochromocytoma and neuroblastoma for which meta-iodobenzylguanidine therapy is 

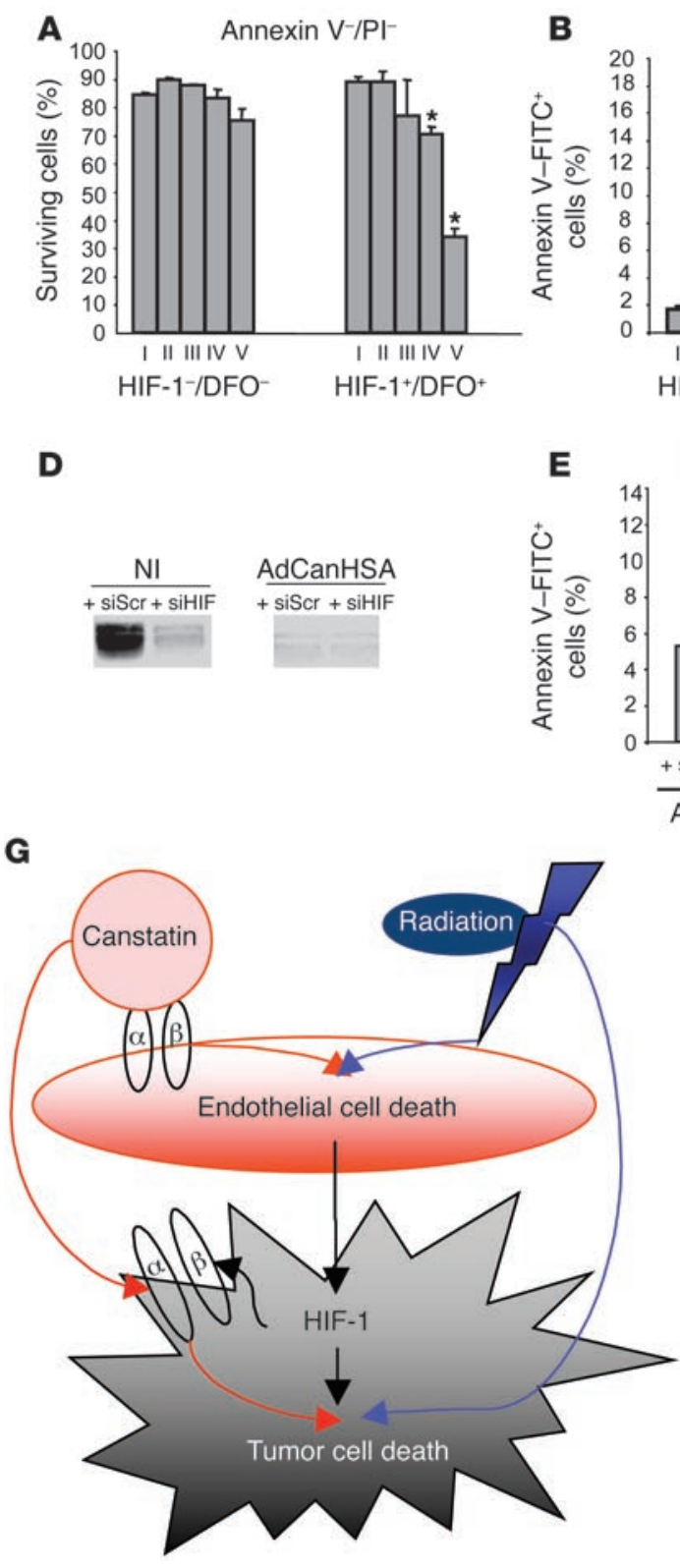

Annexin $\mathrm{V}^{+} / \mathrm{PI}^{-}$

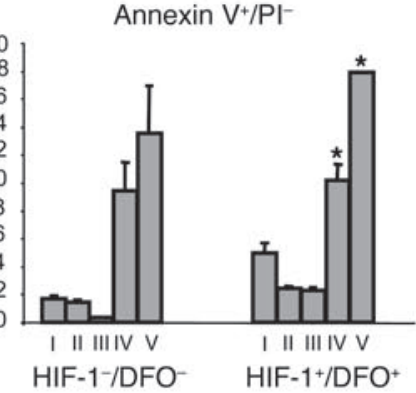

Annexin $\mathrm{V}^{+} / \mathrm{Pl}^{-}$

$\mathrm{HIF}-1^{+} / \mathrm{DFO}^{+}$

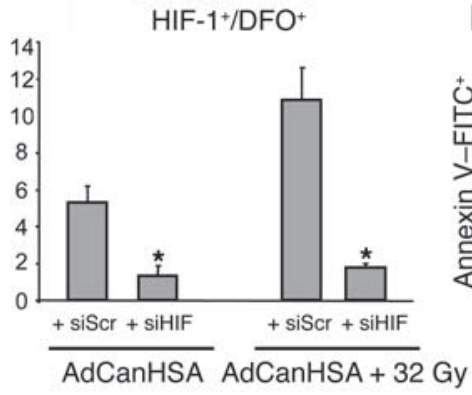

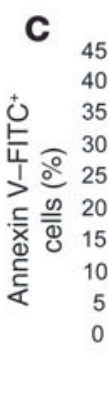

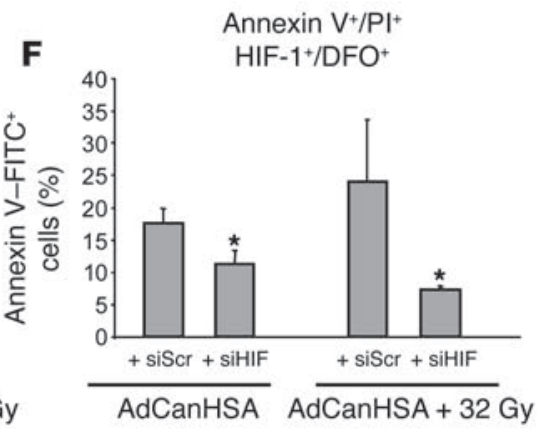

\section{Figure 6}

HIF-1 is the key regulator of the tumor apoptotic switch following combined therapy. FACS, annexin V-FITC, and propidium iodide [PI] profiles of MDA-MB-231 cells that were viable (A); in early apoptosis with membrane integrity $(\mathbf{B})$; or in late apoptosis with death following AdCanHSA infection combined or not with $\gamma$-radiation (C). (D) To assess the suppression of HIF-1 $\alpha$ expression, Western blotting was done on noninfected or AdCanHSA-infected MDA-MB-231 cells that were transfected with random siRNA (siRNA scrambled [siScr]) or siRNA HIF-1 $\alpha$ (siHIF). (E and F) Annexin V-FITC staining was performed on transfected MDA-MB-231 cells following AdCanHSA infection combined or not with $\gamma$-radiation. Bars represent SEM. Each experiment was done twice. ${ }^{*} P<0.05$. (G) Schematic illustration of the role of HIF-1 in tumor response to canstatin and/or radiation. Endothelial cell death is directly triggered by both canstatin through an integrin-mediated mitochondrial apoptotic mechanism and radiation exposures through an apoptotic pathway. The resulting microvascular dysfunction induces $\mathrm{HIF}-1 \alpha$ expression, as a mandatory signal regulating the tumor adaptive response. HIF-1 $\alpha$ in turn upregulates integrin-receptor expression on tumor cell surface, enhancing specifically HIF- $1 \alpha$ signaling tumor apoptotic pathway triggered by canstatin. Moreover, HIF-1 confers the conversion of sublethal radiation damage, such as mitotic arrest and tetraploid status, into lethal lesions through aberrant mitosis in both endothelial and tumor cells. Collectively, induction of profound tumor cell death requires both canstatininduced tumor apoptotic pathway and radiation-mediated mitotic catastrophe through an HIF-1 signaling molecular mechanism.

ineffective, to endocrine tumors treated with radiolabeled somatostatin analogs, and even in patients treated with radiolabeled monoclonal antibodies or external beam radiation therapy, for which the radiation dose applied might be suboptimal (38).

Angiogenic inhibitors also improve the therapeutic index of external beam radiation therapy (16-22). Nevertheless, the mechanisms underlying enhanced radiation response caused by antiangiogenic agents require further elucidation. Radiation therapy alone is known to trigger tumor resistance through increased HIF-1 activity, leading to upregulation of angiogenesis as well as tumor cell survival pathways $(13,15,18)$. HIF-1 is a key cellular modulator that is shared by many different signaling pathways, such as apoptosis, which has proven to be tightly interwoven with other essential cell pathways $(23,24)$.

Here, we show that endothelial cells are the first target of both canstatin and radiation. The signaling pathway leading to endothelial cell death is not dependent on HIF-1. In contrast, tumor cell death is a secondary event that depends on both endothelial cell death and HIF-1 activity. For the first time to our knowledge, we show in vivo and in vitro that combined therapy induces a marked inhibition of tumor vascularization that triggers HIF-1 expression, which then drops over the treatment period, whereas the antitumor activity of canstatin increases through an HIF-1-dependent tumor apoptotic pathway (Figure 6G). Thus, combined therapy can overcome HIF-1mediated survival mechanisms induced by radiation therapy itself or angiogenesis inhibition therapy alone $(18,20,21)$. Experimental studies have previously shown that decreasing HIF-1 activity in tumors can reduce tumor growth, suggesting that antagonizing the HIF-1 signaling survival pathway could help in the treatment of cancer (24). In contrast, we establish here that HIF-1 activation is important in driving tumor cell death induc- 
tion. Indeed, in vitro, HIF-1 transcription was not downregulated by canstatin. Moreover, since in vitro only high concentrations of HIF-1 were shown to lead to canstatin-induced apoptotic tumor cell death (Figure 4C), the strength of HIF-1 expression might determine whether cells become apoptotic or adapt to hypoxia and survive. Severe hypoxia induced by combined therapy might initiate apoptosis, whereas tumor cells adapt to mild hypoxia and survive following canstatin alone or radiation alone.

More precisely, the cell-surface receptor triggering the HIF-1 signaling apoptotic pathway was characterized. As we showed previously, the binding of canstatin to $\alpha_{v} \beta_{3}$ and $\alpha_{v} \beta_{5}$ integrins induces both endothelial and tumor cell apoptosis, thereby eliminating the codependent cycle of tumor growth and angiogenesis (29). Our present findings show that the HIF-1-mediated tumor apoptotic pathway is triggered through the binding of canstatin to $\alpha_{v} \beta_{5}$ integrins, whose cell-surface expression is strongly increased by HIF-1 (Figure 6G). In contrast, HIF-1 activity is not essential in endothelial cells to amplify the apoptotic pathway because integrins usually highly expressed on endothelial cell surfaces are only modestly increased following HIF-1 expression. Given the previous studies showing that ROSs induce tumor HIF-1 activity $(15,39,40)$, which can control tumor cell adhesion, migration, and invasion through regulation of a key integrin expression (41), antagonists of $\alpha_{v} \beta_{5}$ integrins, such as canstatin, hold a definite therapeutic advantage by blocking the HIF-1 signaling survival pathway generated by irradiated tumor tissue.

Several molecular mechanisms are known to block tumor cell proliferation after irradiation to ensure genomic integrity: cells may either undergo cell-cycle arrest with DNA repair or succumb to related forms of programmed cell death. Given that radiationinduced ROS accumulation leads to $\operatorname{HIF-1}$ stabilization $(15,40)$, we further delineated the role of HIF-1 on cellular radiosensitivity, focusing the study on both its apoptotic and mitotic potential. Our observations indicate that radiation-induced mitotic arrest and tetraploidy in both endothelial and tumor cells are not lethal by themselves. Disrupted passage through mitosis often leads to the production of aneuploid progeny, which may confer a proliferative advantage, as previously described $(32,33)$. Interestingly, HIF- 1 allows cells to escape from $\mathrm{G}_{2} / \mathrm{M}$ cell-cycle arrest, both forcing them to enter mitosis prematurely before DNA damage has been repaired and impeding tetraploidization. It has been reported that a weakened checkpoint signal is sufficient to maintain cell viability but leads to aneuploidy. Also, a completely inactive mitotic checkpoint results in cell-autonomous lethality (42). Thus, a large proportion of aberrant mitoses induced by HIF-1 may become lethal by mitotic catastrophe $(34,43)$. Conversely, suppression of mitotic catastrophe leads to aneuploidy, and chromosomal instability is linked to the progressive development of high-grade, invasive tumors $(33,43)$. Mitotic catastrophe is functionally linked to apoptosis, but p53 is not involved in mitotic catastrophe $(43,44)$, confirming that this mechanism may be active both in p53 WT endothelial cells or p53-null tumor cell lines. Nevertheless, p53 status has the intrinsic capacity to eliminate tetraploid cells and suppress the proliferative advantage (33).

Our study highlights an important connection between radiation and antiangiogenic treatments that could potentially be taken advantage of to overcome the HIF-1-dependent tumor radioresistance in clinical practice.

\section{Methods}

\section{Recombinant adenoviruses}

AdNIS and AdCanHSA are $\Delta \mathrm{E} 1-\Delta \mathrm{E} 3$ recombinant adenoviruses expressing, respectively, the rat NIS and the human canstatin, fused to HSA moiety, as previously described $(9,29)$. AdCO1, a negative control, is an empty E1E3-deleted recombinant adenovirus with no transgene replacing the E1 genes (17).

\section{Cell culture}

HUVECs and mammary MDA-MB-231 cells have been previously described (29). RCC4 cells, known to harbor a VHL mutation (VHL mutated), ablating proteolytic regulation of HIF-1, and RCC cells stably transfected with WT pVHL (VHL WT) were grown in DMEM supplemented with $10 \%$ FBS (45).

For hypoxic treatment, cells were grown in the presence of $1,10,50$, or $100 \mu \mathrm{mol} / \mathrm{l}$ of DFO (Sigma-Aldrich).

\section{Tumor models and in vivo iodide experiments}

Xenografted MDA-MB-231 tumor mouse model. All in vivo experiments were approved by the Direction des Services Veterinaires du Val de Marne, Rungis, France.

Human tumors were induced by s.c. injection of $4 \times 10^{6}$ MDA-MB-231 cells into the dorsa of nu/nu mice. At day 1 , when tumor volumes had reached a mean value of $72 \pm 5 \mathrm{~mm}^{3}$, the animals were randomized into 4 groups ( $n=9$ per group). All tumors received 2 injections, 72 hours apart (days 1 and 4 ), of $4 \times 10^{9} \mathrm{PFU}$ of adenoviruses per intratumor injection. Each injection contained 2 adenoviruses $\left(2 \times 10^{9} \mathrm{PFU}\right.$ per adenovirus): AdCO1 and AdNIS (group 2), AdCO1 and AdCanHSA (group 3), or AdNIS and AdCanHSA (group 4). The control group (group 1) received $4 \times 10^{9}$ PFU of AdCO1 per injection.

${ }^{123}$ I and ${ }^{131}$ I were injected i.p., at 24 hours (day 5) or 48 hours (day 6) respectively, after the second adenoviral injection. During the week before iodide administration, thyroxine treatment was given to suppress thyroid iodide uptake (9).

Blood samples were collected at day 10 to quantify CanHSA protein levels by ELISA (29). For uptake measurements ex vivo, mice were sacrificed at 4, 8, and 24 hours following the injection of $300 \mu \mathrm{Ci}$ of ${ }^{131} \mathrm{I}$. Tumors were weighed, and radioactivity was quantified using a calibrated well gamma counter (Compugamma 1282; LKB Wallac). For imaging experiments, animals were anesthetized 4 hours following the injection of $50 \mu \mathrm{Ci}$ of ${ }^{123} \mathrm{I}$. Scintigraphy was performed with a gamma camera (Axis; Philips Medical Systems). The amount of radioactivity present in each tumor was quantified using ImageJ software for computer-assisted image analysis (version 1.31; http://rsb.info.nih.gov/ij.). For radioiodide therapy, mice were injected with $100 \mu \mathrm{Ci}$ (data not shown) or $300 \mu \mathrm{Ci}$ of ${ }^{131} \mathrm{I}$. To evaluate treatment efficacy, tumor sizes were monitored every week for 4 weeks following adenoviral injections. The tumors were removed and fixed in FineFIX (Milestone) at days 8 and 20 after injection of radioiodide for immunohistological analyses.

TRP-1 transgenic mouse model. This transgenic mouse line developed tumors in the RPE and in the choroid-retinal epithelium interface (46). TRP-1 mice were injected i.v. with $10^{9} \mathrm{PFU}$ of AdCanHSA or AdCO1, both 3 days after birth in the temporal vein and 15 days after birth in the retroorbital vein. At day 21 after birth, a single intravitreal local injection of AdNIS or AdCO1 (109 PFU) was performed within both ocular eyeballs in each mouse. Iodide was injected i.p. 72 hours following the local injection of AdNIS or AdCO1 ( $n=10$ per group).

A single dose of ${ }^{131} \mathrm{I}$ was administered for uptake measurement ex vivo $(50 \mu \mathrm{Ci})$ or for therapy $(300 \mu \mathrm{Ci})$. Heads of TRP-1 mice were removed 
45 days after birth (i.e., 21 days after radioiodide treatment) and fixed in FineFIX for immunohistological analyses.

\section{Quantification of CanHSA in vivo}

CanHSA protein in sera of xenografted mice was quantified by ELISA, as previously described (29).

\section{Histology and immunobistochemistry}

MDA-MB-231 xenografted tumors. After fixation of tumors in FineFIX, paraffin sections were stained with hematoxylin-eosin-saffranin (HES).

Measurements of TRP-1 tumor area. The heads of TRP-1 mice were fixed in FineFIX and embedded in paraffin. Five-micrometer sections of eyeballs were prepared for each animal and stained with HES. Tumor areas were drawn and measured with PixCyt, a software package for computer-assisted image analysis, as previously described (47).

Analysis of NIS and CanHSA expression. NIS protein and CanHSA expression protein were revealed by immunohistochemistry using anti-NIS (9) and anti-HSA (Sigma-Aldrich) polyclonal antibodies, respectively, and then revealed with the DAB PowerVision kit (ImmunoVision Technologies Co.) (data not shown).

Quantification of intratumor microvessels. Histological slides from xenografted animals were incubated with a monoclonal rat anti-mouse CD34 antibody (Hycult Biotechnology). For signal amplification, slides were then incubated with rabbit anti-rat immunoglobulins (Dako), and the signal was revealed with the rabbit DAB PowerVision kit (ImmunoVision Technologies Co.).

A posterior section of the head of each transgenic animal was incubated with TRITC-conjugated lectin antibody (Sigma-Aldrich).

CAIX expression. The samples were incubated with a monoclonal antihuman CAIX antibody (MN antigen; Slovak Academy of Sciences, Bratislava, Slovak Republic). The cytoplasmic signal was revealed with the DAB HistoMouse-Max kit (Zymed Laboratories Inc.).

Caspase-3 and -9 expression. MDA-MB-231-derived tumors were immunostained for polyclonal rabbit anti-human cleaved caspase- 3 and caspase-9 (BioValley).

Quantitative digital analysis of tumor tissue sections. Each slide was analyzed using a Zeiss Axiophot microscope. A single representative whole tumor tissue section from each animal was digitized using a slide scanner (Nikon). Prior to computerized analysis, regions of interest were drawn to exclusively select tumor tissue and to eliminate both nontumor tissue and large colored artifact deposits. Quantification of CAIX-, caspase-3- and caspase-9-stained tumor cell surfaces and CD34-stained blood vessels on histological slides was achieved using PixCyt software (47). The ratio between stained and unstained tumor cells was then established.

Apoptotic cell quantification. An in situ cell death detection kit (Roche Applied Science) employing the TUNEL method has been previously described (29).

HIF-1 analysis. To quantify HIF-1 $\alpha$ mRNA levels within treated MDA-MB-231 tumors described above, paraffin sections were incubated with a ${ }^{32}$ P-labeled HIF- $1 \alpha$ antisense probe, and in situ hybridization was performed as previously described (48).

\section{Western blot analysis}

In vivo, for HIF- $1 \alpha$ protein expression analysis, MDA-MB-231 xenografted tumors were dissected at day 20 following ${ }^{131}$ I injection, as described above. Cytoplasmic and nuclear protein extracts were incubated with

monoclonal mouse anti-HIF-1 $\alpha$ (BD Biosciences - Pharmingen) and were revealed with a peroxidase-conjugated goat anti-mouse antibody (Jackson ImmunoResearch Laboratories Inc.).

In vitro, HUVECs, MDA-MB-231 and RCC (RCC4) cells were harvested at the indicated time points after infection with adenovirus. HIF- $1 \alpha$, cleaved caspase-3, and ERK expression was analyzed using anti-human HIF-1 $\alpha$, anti-human cleaved caspase-3 (described above), or anti-ERK antibodies (Upstate).

Staurosporine was used in order to induce apoptosis, as previously described (29).

\section{Flow cytometry}

Cell-cycle studies. In vitro, HUVECs, MDA-MB-231 and RCC (RCC4) cells, grown with or without DFO $(100 \mu \mathrm{mol} / \mathrm{l})$, were irradiated with $1.824 \mathrm{~Gy} / \mathrm{min}$ ${ }^{137} \mathrm{Cs} \gamma$-rays at different doses. Cells were collected 48 hours after irradiation. Cells were suspended in PBS and then fixed with $70 \%$ ethanol at $4{ }^{\circ} \mathrm{C}$. After centrifugation, the supernatants were discarded, and cellular DNA was stained in a solution containing $10 \mu \mathrm{mol} / 1$ of DAPI.

Measurement of apoptotic cell death. In vitro, MDA-MB-231 cells, grown with or without DFO $(1,10,50$, or $100 \mu \mathrm{mol} / \mathrm{l})$ were harvested 48 hours following adenoviral infection and/or irradiation (coupled to or in the absence of siRNA). Apoptotic cells were immunostained with Annexin VFITC Apoptosis Detection Kit I, as recommended by the manufacturer (BD Biosciences - Pharmingen). Data analysis was performed with CellQuest software (version $3.4 ; \mathrm{BD}$ ).

\section{HIF-1 $\alpha$ and integrin gene silencing in MDA-MB-231 cells}

siRNA $\beta 3$ and $\beta 5$ and siRNA HIF-1 were purchased from Ambion and Eurogentec, respectively, as previously described $(29,49)$.

\section{Statistics}

Each experiment was done twice. Statistical analysis was performed using 1-tailed Student's $t$ test (unilateral and unpaired). Differences with a $P$ value less than 0.05 were considered statistically significant.

\section{Acknowledgments}

This work was supported by the Centre National de la Recherche Scientifique, the Institut Gustave Roussy, the Commissariat à l'Energie Atomique, Electricité de France, the Ligue Nationale contre le Cancer, and the Association pour la Recherche sur le Cancer. We sincerely thank the Service Commun d'Expérimentation Animale (SCEA) and especially Annie Rouches, Colette Chianale, and Monique Stanciu for animal care. We warmly acknowledge Sylviane Bayet and Frédéric Lavielle for assistance.

Received for publication September 7, 2006, and accepted in revised form April 10, 2007.

Address correspondence to: Claire Magnon, Division of Hematology and Oncology, Mount Sinai Hospital, Box 1079, One Gustave L. Levy Place, New York, New York 10029, USA. E-mail: clairemagnon@ free.fr or claire.magnon@mssm.edu.

Michel Perricaudet and Martin Schlumberger contributed equally to this work.

\footnotetext{
1. Schlumberger, M. 1998. Papillary and follicular thyroid carcinoma. N. Engl. J. Med. 338:297-306.

2. Cooper, D.S., et al. 2006. Management guidelines for patients with thyroid nodules and differentiated thyroid cancer. Thyroid. 16:109-142.

3. Dai, G., Levy, O., and Carrasco, N. 1996. Cloning and

characterization of the thyroid iodide symporter. Nature. 379:458-460.

4. Eskandari, S., et al. 1997. Thyroid Na+/I- sym porter. Mechanism, stoichiometry, and specificity. J. Biol. Chem. 272:27230-27238.

5. Filetti, S., et al. 1999. Sodium/iodide symporter: a key
}

transport system in thyroid cancer cell metabolism. Eur. J. Endocrinol. 141:443-457.

6. Shimura, H., Haragushi, K., Miyazaki, A., Endo, T., and Onaya, T. 1997. Iodide uptake and experimental ${ }^{131}$ I therapy in transplanted undifferentiated thyroid cancer cells expressing the $\mathrm{Na}^{+} / \mathrm{I}^{-}$symport- 
er gene. Endocrinology. 138:4493-4496.

7. Spitzweg, C., et al. 2000. Treatment of prostate cancer by radioiodine therapy after tissue-specific expression of the sodium iodide symporter. Cancer Res. 60:6526-6530.

8. Smit, J.W.A., et al. 2000. Reestablishment of in vitro and in vivo iodide uptake by transfection of the human sodium iodide symporter in a hNIS defective human thyroid carcinoma cell line. Thyroid. 10:939-943.

9. Boland, A., et al. 2000. Adenovirus-mediated transfer of the thyroid sodium/iodide symporter gene into tumors for a targeted radiotherapy. Cancer Res. 60:3484-3492.

10. Dingli, D., et al. 2003. Genetically targeted radiotherapy for multiple myeloma. Blood. 102:489-496.

11. Schipper, M.L., et al. 2003. Radioiodide treatment after sodium iodide symporter gene transfer is a highly effective therapy in neuroendocrine tumor cells. Cancer Res. 63:1333-1338.

12. Garcia-Barros, M., et al. 2003. Tumor response to radiotherapy regulated by endothelial cell apoptosis. Science. 300:1155-1159.

13. Moeller, B.J., Cao, Y., Li, C.Y., and Dewhirst, M.W. 2004. Radiation activates HIF-1 to regulate vascular radiosensitivity in tumors: role of reoxygenation, free radicals, and stress granules. Cancer Cell. 5:429-441.

14. Gorski, D.H., et al. 1999. Blockage of the vascular endothelial growth factor stress response increases the antitumor effects of ionizing radiation. Cancer Res. 59:3374-3378.

15. Abdollahi, A., et al. 2003. SU5416 and SU6668 attenuate the angiogenic effects of radiationinduced tumor cell growth factor production and amplify the direct anti-endothelial action of radiation in vitro. Cancer Res. 63:3755-3763.

16. Mauceri, H.J., et al. 1998. Combined effects of angiostatin and ionizing radiation in antitumor therapy. Nature. 394:287-291.

17. Griscelli, F., et al. 2000. Combined effects of radiotherapy and angiostatin gene therapy in glioma tumor model. Proc. Natl. Acad. Sci. U. S. A. 97:6698-6703.

18. Lee, C.G., et al. 2000. Anti-vascular endothelial growth factor treatment augments tumor radiation response under normoxic or hypoxic conditions. Cancer Res. 60:5565-5570.

19. Salloum, R.M., et al. 2000. NM-3, an isocoumarin, increases the antitumor effects of radiotherapy without toxicity. Cancer Res. 60:6958-6963.

20. Geng, L., et al. 2001. Inhibition of vascular endothelial growth factor receptor signaling leads to reversal of tumor resistance to radiotherapy. Cancer Res. 61:2413-2419.

21. Griffin, R.J., et al. 2002. Simultaneous inhibition of the receptor kinase activity of vascular endothelial, fibroblast, and platelet-derived growth factors suppresses tumor growth and enhances tumor radiation response. Cancer Res. 62:1702-1706.

22. Gorski, D.H., et al. 2003. Prolonged treatment with angiostatin reduces metastatic burden during radiation therapy. Cancer Res. 63:308-311.

23. Carmeliet, P., et al. 1998. Role of HIF-1a in hypoxiamediated apoptosis, cell proliferation and tumour angiogenesis. Nature. 394:485-490.

24. Harris, A.L. 2002. Hypoxia - a key regulatory factor in tumour growth. Nat. Rev. Cancer. 2:38-47.

25. Greijer, A.E., and Van der Wall, E. 2004. The role of hypoxia inducible factor 1 (HIF-1) in hypoxia induced apoptosis. J. Clin. Pathol. 57:1009-1014.

26. Friedlander, M., et al. 1995. Definition of two angiogenic pathways by distinct $\alpha_{v}$ integrins. Science. 270:1500-1502.

27. Kamphaus, G.D., et al. 2000. Canstatin, a novel matrix-derived inhibitor of angiogenesis and tumor growth. J. Biol. Chem. 275:1209-1215.

28. Panka, D.J., and Mier, J.W. 2003. Canstatin inhibits Akt activation and induces Fas-dependent apoptosis in endothelial cells. J. Biol. Chem. 278:37632-37636.

29. Magnon, C., et al. 2005. Canstatin acts on endothelial and tumor cells via mitochondrial damage initiated through interaction with $\alpha_{v} \beta_{3}$ and $\alpha_{v} \beta_{5}$ integrins. Cancer Res. 65:4353-4361.

30. Spitzweg, C., et al. 2001. In vivo sodium iodide symporter gene therapy of prostate cancer. Gene Ther. 8:1524-1531.

31. Wykoff, C.C., et al. 2000. Hypoxia-inducible expression of tumor-associated carbonic anhydrases. Cancer Res. 60:7075-7083.

32. Kops, G.J., Weaver, B.A., and Cleveland, D.W. 2005. On the road to cancer: aneuploidy and the mitotic checkpoint. Nat. Rev. Cancer. 5:773-785.

33. Fujiwara, T., et al. 2005. Cytokinesis failure generating tetraploids promotes tumorigenesis in $\mathrm{p} 53$ null cells. Nature. 437:1043-1047.

34. Okada, H., and Mak, T.W. 2005. Pathways of apoptotic and non-apoptotic death in tumour cells. Nat. Rev. Cancer. 4:592-603.

35. Tao, W., et al. 2005. Induction of apoptosis by an inhibitor of the mitotic kinesin KSP requires both activation of the spindle assembly checkpoint and mitotic slippage. Cancer Cell. 8:49-59.

36. Bergers, G., Javaherian, K., Lo, K.M., Folkman, J., and Hanahan, D. 1999. Effects of angiogenesis inhibitors on multistage carcinogenesis in mice. Science. 284:808-812.

37. Robbins, R.J., and Schlumberger, M.J. 2005. The evolving role of (131)I for the treatment of differentiated thyroid carcinoma. J. Nucl. Med. 46(Suppl. 1):28S-37S.

38. Wiseman, G.A., and Kvols, L.K. 1995. Therapy of neuroendocrine tumors with radiolabeled MIBG and somatostatin analogues. Semin. Nucl. Med. 25:272-278.

39. Mazure, N., Chen, E.Y., Laderoute, K.R., and Giaccia, A.J. 1997. Induction of vascular endothelial growth factor by hypoxia is modulated by PI3K/ Akt signaling pathway in Ha-ras-transformed cells through a hypoxia inducible factor- 1 transcriptional element. Blood. 90:3322-3331.

40. Chandel, N.S., et al. 2000. Reactive oxygen species generated at mitochondrial complex III stabilize hypoxia-inducible factor-1a during hypoxia. J. Biol. Chem. 275:25130-25138.

41. Cowden Dahl, K.D., Robertson, S.A., Weaver, V.M., and Simon, M.C. 2005. Hypoxia-inducible factor regulates $\alpha_{v} \beta_{3}$ integrin cells surface expression. Mol. Biol. Cell. 16:1901-1912.

42. Weaver, B.A.A., and Cleveland, D.W. 2005. Decoding the links between mitosis, cancer and chemotherapy: the mitotic checkpoint, adaptation and cell death. Cancer Cell. 8:7-12.

43. Castedo, M., et al. 2004. Mitotic catastrophe constitutes a special case of apoptosis whose suppression entails aneuploidy. Oncogene. 23:4362-4370.

44. Taylor, W.R., and Stark, G.R. 2001. Regulation of the G2/M transition by p53. Oncogene. 20:1803-1815.

45. Iliopoulos, O., Kibel, A., Gray, S., and Kaelin, W.G., Jr. 1995. Tumour suppression by the human von Hippel-Lindau gene product. Nat. Med. 1:822-826.

46. Penna, D., Schmidt, A., and Beermann, F. 1998. Tumors of the retinal pigment epithelium metastasize to inguinal lymph nodes and spleen in tyrosinaserelated protein $1 / \mathrm{SV} 40 \mathrm{~T}$ antigen transgenic mice. Oncogene. 17:2601-2607.

47. Elie, N., Plancoulaine, B., Signolle, J.P., and Herlin, P. 2003. A simple way of quantifying immunostained cell nuclei on the whole histological section. Cytometry A. 56:37-45.

48. Le Jan, S., et al. 2003. Angiopoietin-like 4 is a proangiogenic factor produced during ischemia and in conventional renal cell carcinoma. AJP. 162:1521-1528.

49. Berra, E., et al. 2003. HIF prolyl-hydroxylase 2 is the key oxygen sensor setting low steady-state levels of HIF-1 $\alpha$ in normoxia. EMBO J. 22:4082-4090. 